\title{
Emergency department admission revolution during the COVID-19 pandemic: burden or opportunity?
}

\author{
Alessandro Jachetti ${ }^{1}\left[\right.$ Christian Bracco $^{2} \cdot$ Luigi Fenoglio $^{2}$
}

Received: 1 April 2021 / Accepted: 26 April 2021 / Published online: 15 May 2021

(C) Società Italiana di Medicina Interna (SIMI) 2021

Morello et al. performed a multicenter, retrospective, crosssectional study involving some general emergency departments (EDs) in Turin, Italy, from January to August 2020, assessing the impact of the COVID-19 pandemic on ED flows and admissions. Worldwide, the admissions to most EDs decreased according to the evolution of the pandemic; however, there was an increased mortality not only related to the direct impact of COVID-19 on the frail population but also to an excess all-cause mortality due to reduced access to healthcare facilities, overloaded emergency medical services, and the lack of screening and early diagnostics for noncommunicable diseases. Morello et al. highlighted that during the first wave peak, there were not only fewer traumatic injuries but also significantly fewer non-COVID-19 diagnoses compared to 2019 , which were reduced by $80.8 \%$ for neurological diseases [1]. After the first wave, diagnoses of oncological, metabolic/endocrine, and hematological diseases were statistically unchanged; however, in the early, mid, and late periods of the pandemic and all postwave periods, admissions for psychiatric, neurological, non-COVID-19 respiratory, gastrointestinal, urological, and obstetrical/gynecological disease as well as trauma were significantly reduced.

As a result of the pandemic, the changes in ED visits and hospital admissions observed in Morello's work are similar to that described in other reports. The health and social changes observed in a period of a few months that would never have been predicted may explain these changes. One of the reasons for the reduction of admissions is probably the fear of contagion, accentuated by the alarmistic coverage of the pandemic by the media, especially among patients considered to be more frail and at high risk of developing

Alessandro Jachetti

alessandro.jachetti@policlinico.mi.it

1 Fondazione IRCCS Cà Granda Ospedale Maggiore Policlinico Milano, Milano, Italy

2 Azienda Ospedaliera S Croce e Carle Cuneo, Cuneo, Italy severe forms of SARS-CoV2 infection. This element may have influenced those with potentially nonserious situations, who in the pre-COVID-19 era probably made inappropriate use of the ED, to stay home; but it probably also persuaded those with potentially very serious conditions such as coronary syndromes, for whom a significant reduction in diagnoses was observed during the first lockdown period, to avoid treatment.

The reorganization of the ED and hospitals in general to not allow assistance from caregivers and the need to remain isolated has probably affected access. The fear of not being able to see their loved ones for long periods may have led many patients to fear admission to the ED and possible hospitalization. In this regard, the social reorganization that has seen many people forced to stay at home working remotely or out of work may have allowed them to care for their sick loved ones, which was perhaps not possible when everyone was obligated to stay out of the house for most of the day.

Distancing measures, the use of protective equipment, and possible improvements in air quality may also have helped to contain the spread of problems such as respiratory flare-ups and infections of the gastrointestinal tract, which are particularly frequent in the pediatric population. In some situations of chronic pathologies, like diabetes, the implementation of alternative methods of surveillance, such as telemedicine, may have been useful in intercepting destabilization at home at an early stage. In addition, the introduction of treatment strategies may have limited the requirement of ED and hospitalization admissions.

The significant reduction in the number of elective surgical operations may also have contributed to the decreased numbers of admissions and hospitalizations. Since there were fewer surgeries, fewer complications such as infections or thrombotic events occurred.

On the other hand, in some patients, such as diabetic patients, the negative changes in eating habits and sports practice during lockdown may have led to complications 
such as a worsening of glycemic compensation, which requires admission to the ED and consequent hospitalization.

Many people with chronic multi-pathological conditions are among the most frequent users of the ED. They may have been infected with SARS-CoV2 and admitted to the COVID area for respiratory problems or died at home or in a nursing home, thus contributing to a reduction in ED admissions for other problems linked to their basic pathologies.

With regard to the reduction of admissions, there may have been a tendency by doctors themselves to avoid admissions, as they were aware of how the forced isolation in hospital of frail patients could have resulted in very unfavorable prognostic outcomes. We all have seen how the lack of usual caregivers in hospital has contributed to patients developing the feeling of profound abandonment in addition to creating a situation of intense loneliness and despair, which also often seems to promote the development of an unfavorable course.

The work by Morello et al. leads us to consider how the pandemic may have revealed the inadequate use of hospital resources as well as the lack of sensitivity of the population towards pathologies with a high evolutionary risk such as coronary syndromes, since everyone was focused on the fear of SARS-CoV2 infection. On the other hand, it uncovers how the implementation of new treatment modalities in selected categories of patients, such as telemedicine, may represent a useful tool to reduce ED admissions and, in some cases, hospital admissions. This is important because many EDs were divided into a huge "COVID area" and a small "non-COVID area," with overcrowding and long wait times for non-COVID patients.

We can also reflect on the fact that a family system completely absorbed in the work activities of young people, in which the culture of care and the acceptance of death are distorted compared to those of our grandparents, really clashes too much with the social situation of western countries, where the average age of the population is always increasing. Consequently, the prevalence of frail people with polypathological conditions who would probably benefit from care in alternative settings to the hospital is rising.

At this particular moment in history, we have the opportunity to reflect on these aspects and to try, from a completely new point of view, to identify new ways of treating our patients, perhaps starting from the construction of a more solid system of links between the hospital and the local area.

\section{Declarations}

Conflict of interest The authors declare that they have no conflict of interest.

Human and animal rights Authors declare that due to the commentary format, no specific review by Ethical Board has been requested.

Informed consent No patients has been involved in any form of investigation neither data collection.

\section{References}

1. Morello F, Bima P, Ferreri E, Chiarlo M, Balzaretti P, Tirabassi G, Petitti P, Aprà F, Vallino D, Carbone G, Pivetta EE, Lupia E. After the first wave and beyond lockdown: long-lasting changes in emergency department visit number, characteristics, diagnoses, and hospital admissions. Intern Emerg Med. 2021;8:1-8. PMID: 33683538; PMCID: PMC793827310. https://doi.org/10.1007/ s11739-021-02667-2

Publisher's Note Springer Nature remains neutral with regard to jurisdictional claims in published maps and institutional affiliations. 